\title{
Remote Preparation of a Qudit Using Maximally Entangled States of Qubits
}

\author{
Chang-shui $\mathrm{Yu}^{1}, \mathrm{He}$-shan $\mathrm{Song}^{1}$ * and Ya-hong Wang ${ }^{1,2}$ \\ ${ }^{1}$ Department of Physics, Dalian University of Technology, \\ Dalian 116024, P. R. China and \\ ${ }^{2}$ School of information science and engineering, \\ Dalian Institute of Light Industry, \\ Dalian 116034, P. R. China
}

(Dated: November 24, 2017)

\begin{abstract}
Known quantum pure states of a qudit can be remotely prepared onto a group of particles of qubits exactly or probabilistically with the aid of two-level Einstein-Podolsky-Rosen states. We present a protocol for such kind of remote state preparation. We are mainly focused on the remote preparation of the ensembles of equatorial states and those of states in real Hilbert space. In particular, a kind of states of qudits in real Hilbert space have been shown to be remotely prepared in faith without the limitation of the input space dimension.

PACS numbers: 03.67.Hk, 03.65.Bz
\end{abstract}

\section{INTRODUCTION}

Quantum teleportation, as a surprising discovery in quantum information theory by Bennett et al [1], is the teleportation of an unknown quantum state from one place to another without physically sending the particle. E.g. If Alice and Bob share an Einstein-Podolsky-Rosen (EPR) pair, Alice can teleport a qubit to Bob by first carrying out a Bell-state measurement on the qubit and one particle of the EPR pair, then sending two bits of classical information to Bob, who in turn can perform a corresponding unitary operation on his particle (the other particle of the EPR pair) to get the state Alice wants to teleport. As a similar case, remote state preparation (RSP) can be understood as " the teleportation of a known quantum state". However, the difference between them are in that, 1) in RSP Alice knows the state that she wants Bob to prepare, in particular, Alice need not own the state, but only know the information about the state, while in teleportation Alice must own the teleported state, but she need not know the state; 2) in RSP, the required resource can be traded off between classical communication cost and entanglement cost while in quantum teleportation, two bits of forward classical communication and one ebit of entanglement (an EPR pair) per teleported qubit are both necessary and sufficient, and neither resource can be traded off against the other $[2,3]$.

RSP has attracted many attentions in recent years [311]. Bennett et al have generalized RSP for arbitrary qubits, higher dimensional Hilbert spaces and also of entangled systems [3]. The exact and minimal resource consuming RSP protocol is generalized to higher dimension by Zeng and Zhang [4]. Ming-Yong Ye et al consider the faithful remote state preparation using finite classical bits and a non-maximally entangled state [3]. P. Agrawal et al [5] discuss the exact remote state preparation protocol of

*Electronic address: hssong@dlut.edu.cn special ensembles of qubits at multiple locations and also present generalization of their protocol for higher dimensional Hilbert space systems for multi-parties. Berry and Sanders [6] has studied remote preparation of ensemble of mixed states. Simultaneously, RSP for qubits [7] have been implemented in experiment [8,9]. For RSP protocols in higher dimensional Hilbert space, one should note that the analogue of singlet EPR pair (multi-level EPR pair) including the counterpart in Ref. [3] is necessary. However, the distinct disadvantage is that multi-level EPR pairs is very difficult to prepare. On the contrary, the preparation of the EPR pairs can be realized by different schemes $[12,13]$. A more natural question is that, since a state of qudit can be teleported on a group of particles of qubits [14], whether the similar version is suitable for RSP.

In this paper, we present protocols to remotely prepare some qudits mainly including the ensembles of the equatorial states and those of the states in real Hilbert space exactly or probabilistically, using EPR pairs. The key to successfully implementing RSP is that the two states in different Hilbert spaces (a lower dimensional space and a higher dimensional one) can be considered to be equivalent provided that the coefficients of one state expanded in the lower dimensional space are the same to those of the other state expanded in some a subspace (The dimension is the same to the former.) of the higher dimensional space. We find that the ensembles of states in real Hilbert space can be remotely prepared exactly, while only ensembles of the equatorial states with equal input and output space dimensions can be remotely prepared exactly. It is interesting that a kind of states of qudits in real Hilbert space can be remotely prepared without the limitation of the input space dimension, which is different from that in Ref. [4]. The paper is organized as follows. In section II, we study the RSP for the ensembles of the equatorial states; In section III, we study the RSP of the ensembles of states in real Hilbert space. The conclusion is drawn in Section IV.

\section{RSP FOR THE ENSEMBLES OF}




\section{EQUATORIAL STATES}

At first, it is necessary to rigorously describe the equivalence of the states in different Hilbert spaces, as mentioned in Introduction and implied in Ref. [14]. In other words, how do we understand a qudit has been prepared onto a group of quantum states of qubits? Consider two Hilbert spaces denoted by $H_{1}$ and $H_{2}$ with dimensions $D_{1}$ and $D_{2}, D_{1}<D_{2}$. A state $\phi_{1}$ defined in $H_{1}$ can be expanded based on $D_{1}$ orthogonal and complete bases with $\alpha_{j}, j=1,2, \cdots D_{1}$, corresponding to the expanded coefficients. Therefore, for the given bases, $\phi_{1}$ can be denoted by

$$
\phi_{1} \longmapsto\left(\alpha_{1}, \alpha_{2}, \cdots, \alpha_{D_{1}}\right) .
$$

Another state $\phi_{2}$ defined in any $D_{1}$-dimensional subspace of $H_{2}$ can also be expanded in the subspace based on $D_{1}$ orthogonal and complete bases, with $\beta_{j}, j=1,2, \cdots D_{1}$ denoting the expanded coefficients. Analogously, $\phi_{2}$ can be given by

$$
\phi_{2} \longmapsto\left(\beta_{1}, \beta_{2}, \cdots, \beta_{D_{1}}\right) .
$$

One can say that $\phi_{1}$ and $\phi_{2}$ are equivalent if and only

$$
\left(\alpha_{1}, \alpha_{2}, \cdots, \alpha_{D_{1}}\right)=\left(\beta_{1}, \beta_{2}, \cdots, \beta_{D_{1}}\right) .
$$

It is obvious that, if there exists such an equivalent state defined in the $2^{n}$-dimensional Hilbert space to a qudit defined in $D_{1}$ dimension, the minimum $n$ is the integer that satisfies $1+\log _{2} D_{1} \geq n \geq \log _{2} D_{1}$.

Now, we begin our protocol for RSP. Here we suppose that Alice wants to remotely prepare a known $s$-level equatorial state at Bob's location with the state written by

$$
|\psi\rangle=\frac{1}{\sqrt{s}} \sum_{j=0}^{s-1} e^{i \varphi_{j}}|j\rangle,
$$

where $|j\rangle$ is the computational basis and $\varphi_{j}$ is real. For this purpose, she needs to share $L$ EPR pairs with Bob, where $L$ is limited by

$$
1+\log _{2} s \geq L \geq \log _{2} s .
$$

In other words, Alice and Bob have constructed $L$ quantum channels every one of which is an EPR pair. The quantum channel can be written by

$$
|\Phi\rangle=\left[\frac{1}{\sqrt{2}}(|00\rangle+|11\rangle)\right]^{\otimes L},
$$

where the EPR pair chosen is $\frac{1}{\sqrt{2}}(|00\rangle+|11\rangle)$. Expanding eq. (2), $|\Phi\rangle$ can be rewritten by

$$
\begin{aligned}
|\Phi\rangle= & \frac{1}{2^{L / 2}}\left(\left(|0\rangle^{\otimes L}\right)_{A} \otimes\left(|0\rangle^{\otimes L}\right)_{B}\right. \\
& +\left(|0\rangle^{\otimes(L-1)}|1\rangle\right)_{A} \otimes\left(|0\rangle^{\otimes(L-1)}|1\rangle\right)_{B} \\
& \left.+\cdots+\left(|1\rangle^{\otimes L}\right)_{A} \otimes\left(|1\rangle^{\otimes L}\right)_{B}\right),
\end{aligned}
$$

where ()$_{A}$ denotes the particles at Alice's location and ()$_{B}$ corresponds to Bob's. Since any number can be expressed in binary form, a given binary number can be written as a decimal one. In this sense, the quantum channel $|\Phi\rangle$ can be considered to be written in binary form. Correspondingly, the channel in decimal form can be given by

$$
\begin{aligned}
|\Phi\rangle= & \frac{1}{2^{L / 2}}\left((|0\rangle)_{A} \otimes(|0\rangle)_{B}+(|1\rangle)_{A} \otimes(|1\rangle)_{B}\right. \\
& \left.+\cdots+\left(\left|2^{L}-1\right\rangle\right)_{A} \otimes\left(\left|2^{L}-1\right\rangle\right)_{B}\right) .
\end{aligned}
$$

Therefore, $|\Phi\rangle$ can be considered as a $2^{L}$-level EPR pair. In principle, Alice is able to perform any unitary transformation and measurement on the composite system of the $L$ particles at her location. Due to $s \leq 2^{L}$, the equatorial state to be prepared can be expanded in any subsystem of the $2^{L}$ dimensional space. Without loss of the generality, one can choose the subsystem expanded by the basis vectors $\left\{|0\rangle_{A},|1\rangle_{A}, \cdots,|s-1\rangle_{A}\right\}$. In the subsystem, Alice applies a unitary transformation $U^{\prime}$ on the basis vectors of the subsystem then she obtains

$$
U^{\prime}(|k\rangle)_{A}=\left|\psi_{k}\right\rangle=\frac{1}{\sqrt{s}} \sum_{j=0}^{s-1} e^{i(2 k j \pi) / s} e^{i \varphi_{j}}|j\rangle,
$$

where $\varphi_{0}=0$. Namely, Alice performs a unitary transformation $U$ on her $L$ particles where $U=U^{\prime} \oplus I_{\alpha}$ with $I_{\alpha}$ the identity of $\alpha\left(=2^{L}-s\right)$ dimension. After the transformation, the quantum channel is expressed in form by

$$
|\Phi\rangle=\frac{1}{2^{L / 2}}\left(\sum_{k=0}^{s-1} e^{i \chi_{k}}|k\rangle_{A} \otimes\left|\psi_{k}\right\rangle_{B}+\sum_{k=s}^{2^{L}-1}|k\rangle_{A}|k\rangle_{B}\right),
$$

where $\chi_{k}$ are the corresponding phase factors, and not given explicitly. Note that $|k\rangle$ are written in decimal form. One can change them back to binary form. Now, Alice performs single-particle measurements on her particles in the basis $\{|0\rangle,|1\rangle\}$. It is obvious that if Alice obtains the outcomes $|k\rangle_{A}$ (decimal form), $k \leq s-1$, she has to convey to Bob by classical communication whether to apply the corresponding unitary transformation

$$
\left.V_{k}=\operatorname{diag}\left(1, e^{i(2 k \pi) / s}, e^{i(4 k \pi) / s}\right), \cdots, e^{i(2 k \pi)(s-1) / s}\right) \oplus I_{\alpha}
$$

on his $L$ particles or do nothing. Based on the analysis at the beginning, Alice can believe that the $s$-level quantum state has been remotely prepared on the $L$ twolevel particles at Bob's location. However, if Alice obtains the outcomes $|k\rangle_{A}, k>s-1$, she will have to inform Bob of the failing RSP. The successful probability is $s / 2^{L} \times 100 \%>50 \%$, due to the limitation (1). The number of cbits used should be $\log _{2}(s+1)$ if $s \neq 2^{L}$, otherwise $\log _{2} s$, because it is not necessary for Bob to know which measurement outcome leads to the failure. It is obvious that the state is prepared exactly by the protocol only if $s=2^{L}$. Compared with teleportation, 
the $s$-level quantum state can be exactly teleported by $L$ EPR pairs all the time, but the number of cbits used has to be $\log _{2} s+L$.

\section{RSP FOR THE ENSEMBLES OF STATES IN REAL HILBERT SPACE}

At first, let us recall the RSP protocol proposed in Ref. [4] given in our version, where Alice wants to remotely prepare a qudit defined in real $s$-dimensional Hilbert space which is expressed by

$$
|\psi\rangle=\sum_{j=0}^{s-1} \alpha_{j}|j\rangle
$$

with the matrix notation given by $\Psi=\left[\alpha_{0}, \alpha_{1}, \cdots, \alpha_{s-1}\right]^{T}$, where $\alpha_{j}$ is real, $\sum_{j=0}^{s-1} \alpha_{j}^{2}=1$ and superscript $T$ denotes transpose. The quantum channel, i.e. the maximally entangled state shared by Alice and Bob is

$$
|\Phi\rangle_{A B}=\frac{1}{\sqrt{s}} \sum_{j=0}^{s-1}|j\rangle_{A} \otimes|j\rangle_{B}
$$

For a feasible minimum RSP task, there should exist $s$ unitary operators $V_{j}, j=0,1, \cdots, s-1$, independent of $\Psi$ such that all the vectors given by $V_{j} \Psi$ are orthogonal each other. To realize the RSP, Alice first performs a unitary operation $U$ given by

$$
U=\left[V_{0} \Psi, V_{1} \Psi, \cdots, V_{s-1} \Psi\right]^{T},
$$

on the particle of the channel at her location, then she performs single-particle measurement with respect to the basis $\left\{|j\rangle_{A}\right\}$. If she obtains the outcome $|j\rangle_{A}$, she will inform Bob by classical communication. Bob can perform a corresponding unitary operation $V_{j}^{\dagger}$ on his particle based on the outcome, then he obtains the state $|\psi\rangle$. The RSP is completed.

However, not all the qudits can be remotely prepared according to the minimum RSP. In Ref. [4], the authors have shown that the qudit can be remotely prepared onto another one at Bob's location only if $s=1,2,4,8$. It is worthy of being noted that $s=3$ and $s=5,6,7$ are just the special cases of $s=4$ and $s=8$, respectively, which implies that the quantum channels of RSP for $s=3$ and $s=5,6,7$ are 4-level maximally entangled state and 8level one, respectively. The numbers of cbits used for $s=3$ and $s=5,6,7$ are 2 and 3 , respectively, which are greater than $\log _{2} s$. Hence, they are not included in the so called minimum RSP.

Next, we firstly generalize the protocol mentioned above to the case with $L$ EPR pairs as quantum channels. Alice and Bob have to share $L$ EPR pairs with $1+\log _{2} s \geq L \geq \log _{2} s$. The channel is chosen the same to eq. (2). Consider the decimal form, also eq. (4) can be obtained. Eq. (4) is the same to a $2^{L}$-level maximally entangled state in form. When $s \leq 4$, the state to be remotely prepared can be written as

$$
|\psi\rangle=\sum_{j=0}^{3} \alpha_{j}|j\rangle
$$

where $\alpha_{j}=0$ for $j>s-1$. The quantum channel of the two EPR pairs can be given by

$$
|\Phi\rangle_{A B}=\frac{1}{2} \sum_{j=0}^{3}|j\rangle_{A} \otimes|j\rangle_{B},
$$

where $j$ is the decimal form of $\{00,01,10,11\}$. Alice operates $U=\left[V_{0} \Psi, V_{1} \Psi, \cdots, V_{3} \Psi\right]^{T}$ on her two particles of the channel, where $\Psi=\left[\alpha_{0}, \alpha_{1}, \alpha_{2}, \alpha_{3}\right]^{T}, V_{0}=I$, $V_{1}=\left(\begin{array}{cc}-i \sigma_{y} & 0 \\ 0 & -i \sigma_{y}\end{array}\right), V_{2}=\left(\begin{array}{cc}0 & -\sigma_{z} \\ \sigma_{z} & 0\end{array}\right)$ and $V_{3}=$ $\left(\begin{array}{cc}0 & -\sigma_{x} \\ \sigma_{x} & 0\end{array}\right)$, with $I$ the identity, $\sigma_{i}$ Pauli matrices. After Alice's operation, $|\Phi\rangle_{A B}$ is converted into

$$
|\Phi\rangle_{A B} \rightarrow U|\Phi\rangle_{A B}=\frac{1}{2} \sum_{j=0}^{3}|j\rangle_{A} \otimes V_{j}|\psi\rangle_{B}
$$

Alice performs single-particle measurements on her two particles. If Alice obtains $|00\rangle$ (changed back to binary form), Bob does nothing. If Alice obtains $|01\rangle$, Bob operates $V_{1}^{\dagger}$ on his two particles. The others are analogous. What's more, one will find that $V_{j}^{\dagger}$ can be written in the form of $M_{j} \otimes N_{j}$. Therefore, after Bob receives Alice's outcome, he can operate $M_{j}$ and $N_{j}$ on his two particles, respectively. When $4<s \leq 8$, Alice and Bob have to share three EPR pairs as quantum channel. According to the analogous procedure described above, the corresponding qudit to be prepared can be remotely prepared exactly onto three particles at Bob's location. The unitary operation $V_{j}$ needed are the same to those for 8dimensional RSP in Ref. [4]. However, unlike the case in four dimension, not all $V_{j}, j=1,2, \cdots, 8$, can be decomposed into direct product forms, hence Bob has to perform collective unitary operations on his three particles based on Alice's outcomes. The number of cbits used is $L$ for $s \leq 8$, while that for teleportation is $\log _{2} s+L$. The difference between the present protocol and that in Ref. [4] are mainly in that: In Ref. [4], unitary operations on single particle are only needed, but in the present protocol, collective unitary operations on multiple particles have to be preformed.

It is very interesting that a kind of states in real Hilbert space can also be remotely prepared exactly onto a group of two-level particles without the limitation of the input space dimension.

Case 1. Consider the $s$-level state to be prepared given by eq. (8), $s>8$, which can always be expressed in binary form by

$$
|\psi\rangle=|\psi\rangle_{1,2, \cdots, L}=\sum_{i, j, \cdots, k=0}^{1} \alpha_{i j \cdots k} \underbrace{|i\rangle \otimes|j\rangle \otimes \cdots \otimes|k\rangle}_{L},
$$


where $L$ denotes the number of EPR pairs as the quantum channel and $1+\log _{2} s \geq L \geq \log _{2} s$. Furthermore, $\alpha_{i j \cdots k}=0$ if $i \times 2^{0}+j \times 2^{1}+\cdots+k \times 2^{L-1}>s-1$. From the mathematical form of eq. (9), $|\psi\rangle$ can be considered as a $L$-particle quantum state. In this sense, $|\psi\rangle$ is denoted by $|\psi\rangle_{1,2, \cdots, L}$, the density matrix of which can be given by $\rho_{1,2, \cdots, L}$. From the viewpoint of a multipartite quantum state, one can give the reduced density matrix by tracing over one or several of the $L$ subsystems. As we know, a multi-particle pure state is fully separable if and only if all the reduced density matrices tracing over $L-1$ subsystems are pure. Namely,

$$
\sqrt{2 \sum_{r=1}^{L}\left(1-\operatorname{tr} \rho_{r}^{2}\right)}=0,
$$

where $\rho_{r}$ is the reduced density matrix of the $r$ th subsystem $[15,16]$. If we assume $|\psi\rangle_{1,2, \cdots, L}$ is fully separable, $|\psi\rangle_{1,2, \cdots, L}$ can be written by

$$
|\psi\rangle_{1,2, \cdots, L}=|\psi\rangle_{1} \otimes|\psi\rangle_{2} \otimes \cdots \otimes|\psi\rangle_{L},
$$

where $|\psi\rangle_{i}$ are two-level quantum states, formally given by

$$
|\psi\rangle_{i}=a_{i}|0\rangle+b_{i}|1\rangle, a_{i}^{2}+b_{i}^{2}=1,
$$

$a_{i}$ and $b_{i}$ are determined by eq. (9) and eq. (11), and $\alpha_{i j \ldots k}$ are limited by eq. (10).

Since Alice has known all the information on $|\psi\rangle$, to remotely prepare $|\psi\rangle$ at Bob's location, Alice can select the $i$ th EPR pair shared with Bob and carry out measurement on her particle by projecting onto the basis given by

$$
\begin{aligned}
|\psi\rangle_{i} & =a_{i}|0\rangle+b_{i}|1\rangle \\
|\bar{\psi}\rangle_{i} & =b_{i}|0\rangle-a_{i}|1\rangle .
\end{aligned}
$$

Then Alice informs Bob of her outcomes, and Bob has to perform a corresponding unitary transformation $U_{i}=$ $\left(\begin{array}{cc}0 & -1 \\ 1 & 0\end{array}\right)$ on his particle of the $i$ th EPR pair, if he receives the message $|\bar{\psi}\rangle_{i}$, otherwise do nothing. If the same job is done for all the $L$ EPR pairs, Bob will obtain a genuine two-level $L$-particle state $|\psi\rangle_{1,2, \cdots, L}$ (fully separable), which means that the original $s$-level state of qudit has been exactly remotely prepared onto $L$ twolevel particles. The number of cbits used is $L$. It is very obvious that the key in this case is that the RSP for a qudit has been converted into $L$ RSPs for qubit, meanwhile RSP for the states of qubit in real Hilbert space can be exactly implemented all the time [7].

Case 2. Following eq. (9), since $|\psi\rangle_{1,2, \cdots, L}$ has been considered as an $L$-particle quantum state, we can group the $L$ subsystems into $\left[\frac{L}{2}\right]$ parties, with every two subsystems regarded as a party, where $\left[\frac{L}{2}\right]=\left\{\begin{array}{cc}\frac{L}{2}, & \text { even } L \\ \frac{L+1}{2}, & \text { odd } L\end{array}\right.$.
Without loss the generality, we rewrite $|\psi\rangle_{1,2, \cdots, L}$ as

$$
\begin{aligned}
& |\psi\rangle_{1,2, \cdots,\left[\frac{L}{2}\right]} \\
= & \sum_{i, j, l, m, \cdots, k=0}^{1} \alpha_{i j \cdots k} \underbrace{(|i\rangle \otimes|j\rangle) \otimes(|l\rangle \otimes|m\rangle) \otimes \cdots \otimes|k\rangle}_{L},
\end{aligned}
$$

where $(\cdot)$ denotes a party. If $L$ is an even, the subsystems can be grouped exactly, otherwise there has to be a single subsystem left as a special party at the end. Thus $|\psi\rangle$ can also be regarded as a $\left[\frac{L}{2}\right]$-particle quantum state defined in $\underbrace{4 \times \cdots \times 4}$ dimension for even $L$ and in $\left[\frac{L}{2}\right]$

$\underbrace{4 \times 4 \times \cdots \times 4 \times 2}_{\left[\frac{L}{2}\right]}$ dimension for odd $L$. Therefore, we can draw a conclusion as follows. The original quantum state of qudit can be exactly remotely prepared at Bob's location, if $|\psi\rangle_{1,2, \cdots,\left[\frac{L}{2}\right]}$ is fully separable.

The reason for it is simple. If $|\psi\rangle_{1,2, \cdots,\left[\frac{L}{2}\right]}$ is fully separable, $|\psi\rangle_{1,2, \cdots,\left[\frac{L}{2}\right]}$ can be rewritten as

$$
|\psi\rangle_{1,2, \cdots,\left[\frac{L}{2}\right]}=|\varphi\rangle_{1} \otimes|\varphi\rangle_{2} \otimes \cdots \otimes|\varphi\rangle_{\left[\frac{L}{2}\right]},
$$

where $|\varphi\rangle_{i}$ are two-particle quantum states of qubits (or four-level states) for even $L$, given by

$$
|\varphi\rangle_{i}=a_{i}|00\rangle+b_{i}|01\rangle+c_{i}|10\rangle+d_{i}|11\rangle,
$$

with $a_{i}^{2}+b_{i}^{2}+c_{i}^{2}+d_{i}^{2}=1$; For odd $L,|\varphi\rangle_{i}, i<\left[\frac{L}{2}\right]$, are defined the same to those for even $L$, while $|\varphi\rangle_{\left[\frac{L}{2}\right]}=$ $a_{\left[\frac{L}{2}\right]}|0\rangle+b_{\left[\frac{L}{2}\right]}|1\rangle, a_{\left[\frac{L}{2}\right]}^{2}+b_{\left[\frac{L}{2}\right]}^{2}=1$. Alice firstly divides the $L$ EPR pairs into $\left[\frac{L}{2}\right]$ groups corresponding to $|\varphi\rangle_{i}$. Hence RSP for $|\psi\rangle_{1,2, \cdots,\left[\frac{L}{2}\right]}$ is converted into $\left[\frac{L}{2}\right]$ RSPs for four-level states (a two-level state may be included). Since RSP for the four-level states in real Hilbert space can be exactly implemented as mentioned at the beginning of the section, the original state of qudit can be prepared at Bob's location exactly. The number of cbits used here is also $L$.

Case 3. Following eq. (9) again, if we group the $L$ subsystems into $\left[\frac{L}{3}\right]$ parties, with every three subsystems regarded as a party, where $\left[\frac{L}{3}\right]$ denotes the minimal integer greater than $\frac{L}{3}$. Hence $|\psi\rangle$ can be considered as a $\left[\frac{L}{3}\right]$-particle quantum state denoted by $|\psi\rangle_{1,2, \cdots,\left[\frac{L}{3}\right]}$. Since RSP for the eight-level states in real Hilbert space can be exactly implemented [4], based on the same procedure to that in Case 2, we can also draw the conclusion as follows. The original quantum state of qudit can be remotely prepared exactly at Bob's location, if $|\psi\rangle_{1,2, \cdots,\left[\frac{L}{3}\right]}$ is fully separable. The number of cbits used here is $L$ again.

Case 4 . The above three cases imply that the $L$ subsystems of $|\psi\rangle_{1,2, \ldots, L}$ is divided into some parties, where each party includes the subsystems with the same quantity except the subsystems left in the end. If we divided 
the $L$ subsystems into parties such that every party includes at most three subsystems, we can also obtain a new multi-particle state (denoted by $\Psi$ ) with its subsystems defined in different dimensions which do not exceed 8. One can find that RSP for the original state $|\psi\rangle$ can be successfully implemented, if $\Psi$ regarded as a multiparticle state is fully separable. The reason is that the qudit defined in $s$ dimensions with $s \leq 8$ can be remotely prepared exactly. For a given grouping, the number of cbits used here is $L$. Consider different groupings, the number of cbits used will increase, because Alice has to inform Bob of how she has divided the $|\psi\rangle_{1,2, \cdots, L}$.

In fact, for a given $|\psi\rangle$ to be remotely prepared, Alice can perform any unitary transformation $U_{s}$ on it in mind (The practical transformation on $|\psi\rangle$ is not necessary, because it is not the physical state $|\psi\rangle$ that participates in RSP, but Alice performs some operations on her particles of the quantum channel based on the information of $|\psi\rangle$, which is the difference between RSP and teleportation mentioned in Introduction), which changes the initial $|\psi\rangle$ into $U_{s}|\psi\rangle$. Therefore, the previous RSP of $|\psi\rangle$ has to be changed into the RSP of $U_{s}|\psi\rangle$. However, to realize such a RSP, Alice must inform Bob of what she has done on $|\psi\rangle$, i.e. the information of $U_{s}$ by classical communication. Alternately, Alice and Bob can promise before RSP task that only one or several given $U_{s}$ are allowed to perform on $|\psi\rangle$ by Alice. From the viewpoint that $|\psi\rangle$ can be considered as a multi-particle quantum state $|\psi\rangle_{1,2, \cdots, L}$, it is possible that $U_{s}$ is not a local unitary transformation. Hence, the separability of $|\psi\rangle_{1,2, \cdots, L}$ (including all possible groupings mentioned above) will be changed with $U_{s}$. That is to say, the four cases are dependent on $U_{s}$. Therefore, so long as Alice can find a $U_{s}$ so that $U_{s}|\psi\rangle$ satisfies one of the four cases, she can remotely prepare $|\psi\rangle$ successfully onto a group of twolevel particles at Bob's location. However, as mentioned above, Alice has to inform Bob by classical communication of the $U_{s}$ and her measurement outcomes, which means that the number of cbits used will be increased with the increase of the quantity of $U_{s}$ to be employed.
However, it is not expected that the number of cbits used exceeds that used in teleportation. Hence, the quantity of $U_{s}$ should be restricted before RSP task. In the realistic scenarios, before RSP, Alice and Bob have to make some limitation on the channels and Alice's operations such as arranging and numbering their quantum channels, fixing the concrete form and quantity of the transformations $U_{s}$, restricting the form of groupings, and so on, which can effectively decrease the number of cbit used. At least, Alice can only be allowed to follow the above four cases, which means that $U_{s}$ is not allowed and only a kind of grouping can be considered. In a word, Alice and Bob can increase the limitations to decrease the number of cbits used.

\section{CONCLUSIONS}

We have presented protocols to remotely prepare the ensembles of the "equatorial states" and those of the states in real Hilbert space, using EPR pairs exactly or probabilistically. We find that the ensembles of the $s$ level equatorial states can be remotely prepared exactly by $L$ EPR pairs if the input and output space dimensions are equal, otherwise, they can be prepared with the probability greater than $1 / 2$. The number of cbits used is $\log _{2}(s+1)$ if $s \neq 2^{L}$, otherwise $\log _{2} s$. The ensembles of states in real Hilbert space can be remotely prepared exactly all the time if the input space dimensions are less than or equal to 8 . The number of cbits used is $L$. Some states with the input space dimensions greater than 8 can also be shown to be exactly remotely prepared on a group of two-level particles so long as they satisfy one of the all cases mentioned in Section III.

\section{ACKNOWLEDGEMENT}

This work was supported by the National Natural Science Foundation of China, under Grant Nos. 10575017 and 60472017 .
[1] C. Bennett, G. Brassard, C. Crepeau, R. Jozsa, A. Peres, and W. K. Wooters, Phys. Rev. Lett. 70, 1895 (1993).

[2] Ming-Yong Ye, Yong-Sheng Zhang, and Guang-Can Guo, Phys. Rev. A 69, 022310 (2004).

[3] C. H. Bennett, D. P. DiVincenzo, P. W. Shor, J. A. Smolin, B. M. Terhal, and W. K. Wootters, Phys. Rev. Lett. 87, 077902 (2001).

[4] B. Zeng and P. Zhang, Phys. Rev. A 65, 022316 (2002).

[5] P. Agrawal, P. Parashar, and A. K. Pati, quant-ph/0304006

[6] D. W. Berry and B. C. Sanders, Phys. Rev. Lett. 90, 057901 (2003).

[7] A. K. Pati, Phys. Rev. A 63, 014302 (2001).

[8] X. Peng, X. Zhu, X. Fang, M. Feng, M. Liu, K. Gao, quant-ph/0202004
[9] X. Peng, X. Zhu, M. Liu, K. Gao, quant-ph/0208079

[10] H. K. Lo, Phys. Rev. A 62, 012313 (2000).

[11] D. W. Leung, P. Shor, Phys. Rev. Lett. 90, 127905 (2003).

[12] E. Hagley, X. Maitre, G. Nogues, D. Wunderlich, M. Brune, J. M. Raimond, an S. Hroche, Phys. Rev. Lett. 79, 1 (1997).

[13] Q. A. Tuchette, C. S. Wood, B. E. King, C. J. Myatt, D. Leibfried, W. M. Itano, C. Moroc, and D. J. Wineland, Phys. Rev. Lett. 81, 3631 (1998).

[14] Jindong Zhou and Guang Hou, Phys. Rev. A 64, 012301 (2001).

[15] Pranaw Rungta, V. Bužek, Carlton M. Caves, M. Hillery, and G. J. Milburn, Phy.Rev. A 64, 042315 (2001).

[16] A. R. R. Carvalho, F. Mintert, A. Buchleitner, Phys. 
Rev. Lett. 93, 230501 (2004). 